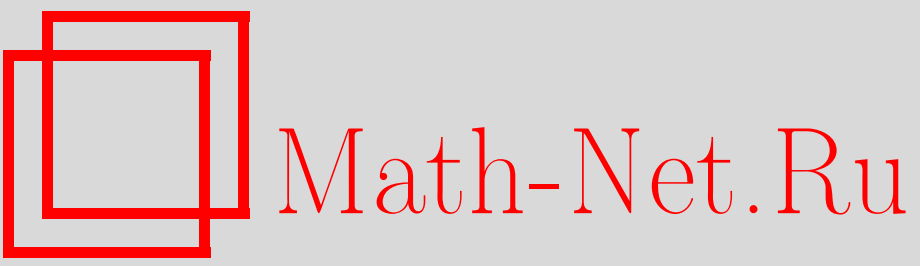

Н. В. Володько, Предельные теоремы для канонических статистик Мизеса и $U$-статистик от $m$-зависимых наблюдений, Теория вероятн. и ее примен., 2010, том 55, выпуск 2, 226-249

DOI: https://doi.org/10.4213/tvp4199

Использование Общероссийского математического портала Math-Net.Ru подразумевает, что вы прочитали и согласны с пользовательским соглашением

http: //www . mathnet.ru/rus/agreement

Параметры загрузки:

IP : 3.82 .47 .9

26 апреля 2023 г., 02:59:52 


\title{
ПРЕДЕЛЬНЫЕ ТЕОРЕМЫ ДЛЯ КАНОНИЧЕСКИХ СТАТИСТИК МИЗЕСА И $U$-СТАТИСТИК ОТ $m$-ЗАВИСИМЫХ НАБЛЮДЕНИЙ ${ }^{1)}$
}

\begin{abstract}
Настоящая работа продолжает исследования, начатые в работах И. С. Борисова, А. А. Быстрова и автора (Сиб. матем. журн., 2006, т. 47, № 6; Матем. труды, 2008, т. 11, № 1; IMS Collections, v. 5), где были доказаны некоторые предельные теоремы для канонических $U$ - и $V$-статистик, построенных по стационарно связанным наблюдениям с условиями $\psi$-, $\varphi$ - или $\alpha$-перемешивания. Однако условия в упомянутых работах, обеспечивающие известное предельное поведение указанных статистик, включают в себя либо весьма существенные ограничения на конечномерные распределения исходной стационарной последовательности, либо некоторые условия регулярности ядер рассматриваемых статистик.

В настоящей работе показано, что в случае стационарных последовательностей $m$-зависимых наблюдений все же возможно обойтись без указанных выше дополнительных условий при описании предельного поведения $U$ - и $V$-статистик.
\end{abstract}

Ключевые слова и фразы: стационарные последовательности случайных величин, $m$-зависимость, ортогональный ряд, канонические $U$ - и $V$-статистики.

1. Введение и предварительные результаты. Рассмотрим стационарную (в узком смысле) последовательность случайных величин $X_{1}, X_{2}, \ldots$, принимающих значения в произвольном сепарабельном метрическом пространстве $\mathfrak{X}$. Обозначим через $F$ распределение $X_{1}$. В дальнейшем нам будет удобно использовать также вспомогательную последовательность $\left\{X_{i}^{*}\right\}$, состоящую из независимых копий случайной величины $X_{1}$.

Напомним определение последовательности $m$-зависимых величин.

\footnotetext{
*Новосибирский государственный университет, Академгородок, ул. Пирогова, 2; Институт математики им. С.Л. Соболева СО РАН, пр-т Академика Коптюга, 4, 630090 Новосибирск, Россия, e-mail: nvolodko@gmail.com

1) Работа выполнена при поддержке Российского фонда фундаментальных исследований (коды проектов 08-01-00962, 09-01-00224), Министерства образования и науки РФ (код проекта РНП.2.1.1.1379), а также программы «Ведущие научные школы» (код проекта НШ-3695.2008.1).
} 
О п р е д е л е н и е 1 . Случайные величины $\left\{X_{i} ; i \geqslant 1\right\}$ называются $m$-зависимыли, если два семейства случайных величин $\left\{X_{i} ; i \leqslant k\right\}$ и $\left\{X_{i} ; i>k+m\right\}$ независимы при любом натуральном $k$.

Пусть $L_{2}\left(\mathfrak{X}^{d}, F^{d}\right)$ обозначает пространство измеримых функций $f\left(t_{1}, \ldots, t_{d}\right)$, заданных на соответствующей декартовой степени пространства $\mathfrak{X}$ и имеющих конечный второй момент по соответствующей продакт-мере, т.е. удовлетворяющих условию $\mathbf{E} f^{2}\left(X_{1}^{*}, \ldots, X_{d}^{*}\right)<\infty$.

О п р е д е л е н и е 2. Функция $f\left(t_{1}, \ldots, t_{d}\right) \in L_{2}\left(\mathfrak{X}^{d}, F^{d}\right)$ называется канонической (или вырожденной), если

$$
\text { E } f\left(t_{1}, \ldots, t_{k-1}, X_{k}, t_{k+1}, \ldots, t_{d}\right)=0
$$

для любого $k$ и для любых $t_{j} \in \mathfrak{X}$.

Определим статистику Мизеса (или $V$-статистику) формулой

$$
V_{n} \equiv V_{n}(f):=n^{-d / 2} \sum_{1 \leqslant j_{1}, \ldots, j_{d} \leqslant n} f\left(X_{j_{1}}, \ldots, X_{j_{d}}\right)
$$

где функция $f\left(t_{1}, \ldots, t_{d}\right)$ (так называемое ядро статистики) является канонической. В случае независимых наблюдений $\left\{X_{i}\right\}$ статистики такого типа (их нередко называют каноническими $V$-статистиками) изучались с середины прошлого века (обзор литературы и конкретные примеры статистик см. в [5]). Впервые предельные теоремы для них были получены в работах Р. Мизеса [10] и В. Хёфдинга [9]. Наряду с $V$ статистиками рассматриваются близкие к (2) функционалы, так называемые $U$-статистики:

$$
U_{n} \equiv U_{n}(f):=n^{-d / 2} \sum_{1 \leqslant i_{1} \neq \cdots \neq i_{d} \leqslant n} f\left(X_{i_{1}}, \ldots, X_{i_{d}}\right) .
$$

Отличие $U$-статистик от $V$-статистик состоит в отсутствии в области суммирования кратных сумм совпадающих индексов (или, как говорят, диагональных подпространств).

Нашей задачей является исследование предельного поведения $U$ - и $V$-статистик, построенных по выборкам растущего объема из последовательности $m$-зависимых случайных величин. В дальнейшем мы полагаем, что $d \geqslant 2$, поскольку в случае $d=1$ упомянутые статистики представляют собой нормированные суммы стационарно связанных случайных величин, для которых соответствующая предельная теория уже разработана.

Метод настоящей работы основан на представлении ядра в виде специального кратного ортогонального ряда. Вкратце напомним классические результаты из этой области, а также основанные на указанном разложении ядра предельные теоремы для канонических $U$ - и $V$-статистик, полученные ранее. 
Поскольку гильбертово пространство $L_{2}(\mathfrak{X}, F)$ также является сепарабельным, в нем существует счетный ортонормированный базис. Более того, положив $e_{0}(t) \equiv 1$, можно методом ортогонализации ГрамаШмидта [4] построить ортонормированный базис $\left\{e_{i}(t)\right\}_{i \geqslant 0}$, содержащий $e_{0}(t)$. Тогда $\mathbf{E} e_{i}\left(X_{1}\right)=0$ для всех значений $i \geqslant 1$ в силу условия ортогональности с $e_{0}(t)$. Условие нормировки означает, что $\mathbf{E} e_{i}^{2}\left(X_{1}\right)=1$ для всех $i \geqslant 1$. Отметим, что при этом семейство функций $\left\{e_{i_{1}}\left(t_{1}\right) e_{i_{2}}\left(t_{2}\right) \cdots e_{i_{d}}\left(t_{d}\right) ; i_{1}, i_{2}, \ldots, i_{d}=0,1, \ldots\right\}$ является ортонормированным базисом в пространстве $L_{2}\left(\mathfrak{X}^{d}, F^{d}\right)$ (см. [4]).

Таким образом, можно разложить ядро $f\left(t_{1}, \ldots, t_{d}\right)$ рассматриваемых статистик по базису $L_{2}\left(\mathfrak{X}^{d}, F^{d}\right)$. Если, кроме того, ядро является вырожденным, то элемент $e_{0}(t) \equiv 1$ не принимает участия в разложении (подробнее см. [2]), т.е. имеет место разложение

$$
f\left(t_{1}, \ldots, t_{d}\right)=\sum_{i_{1}, \ldots, i_{d}=1}^{\infty} f_{i_{1} \ldots i_{d}} e_{i_{1}}\left(t_{1}\right) \cdots e_{i_{d}}\left(t_{d}\right)
$$

где ряд в правой части равенства (4) сходится в норме $L_{2}\left(\mathfrak{X}^{d}, F^{d}\right)$. При этом и само равенство (4) понимается как равенство в $L_{2}\left(\mathfrak{X}^{d}, F^{d}\right)$, т.е. для почти всех относительно продакт-меры $F^{d}$ векторных аргументов $\left(t_{1}, \ldots, t_{d}\right)$.

Остановимся на случае $d=2$. Рассмотрим интегральный линейный оператор с симметричным каноническим ядром $f \in L_{2}\left(\mathfrak{X}^{2}, F^{2}\right)$, переводящий $L_{2}(\mathfrak{X}, F)$ в себя. Поскольку этот линейный оператор будет вполне непрерывным и самосопряженным, то в сепарабельном гильбертовом пространстве $L_{2}(\mathfrak{X}, F)$ существует ортонормированный базис, состоящий из собственных векторов этого интегрального оператора. При этом, в силу вырожденности ядра, функция $e_{0}(t) \equiv 1$ является собственным вектором оператора, соответствующим собственному числу $\lambda_{0}=0$. Тогда для этого базиса представление (4) при $d=2$ будет выглядеть следующим образом:

$$
f\left(t_{1}, t_{2}\right)=\sum_{k=1}^{\infty} \lambda_{k} e_{k}\left(t_{1}\right) e_{k}\left(t_{2}\right)
$$

где $\lambda_{k}$ - собственное число, отвечающее функции $e_{k}(\cdot)$ (см. [2], [5] и [8]).

Таким образом, при замене векторной переменной $\left(t_{1}, \ldots, t_{d}\right)$ на независимые наблюдения $\left(X_{1}^{*}, \ldots, X_{d}^{*}\right)$ частичные суммы ряда в правой части (4) (или (5) при $d=2$ ) сходятся к случайной величине $f\left(X_{1}^{*}, \ldots, X_{d}^{*}\right)$ в среднеквадратичном, а следовательно, и по распределению. Если, к тому же, коэффициенты разложения $\left\{f_{i_{1} \ldots i_{d}}\right\}$ (или, соответственно, $\lambda_{k}$ для $d=2$ ) абсолютно суммируемы, то в силу теоремы Б. Леви и элементарной оценки $\mathbf{E}\left|e_{i_{1}}\left(X_{1}^{*}\right) \cdots e_{i_{d}}\left(X_{d}^{*}\right)\right| \leqslant 1$ ряд в (4) (или (5)) будет 
сходиться к случайной величине $f\left(X_{1}^{*}, \ldots, X_{d}^{*}\right)$ и почти наверное относительно распределения $F^{d}$ вектора $\left(X_{1}^{*}, \ldots, X_{d}^{*}\right)$. Однако при замене аргументов $\left(X_{1}^{*}, \ldots, X_{d}^{*}\right)$ на исходные зависимые $\left(X_{1}, \ldots, X_{d}\right)$ только что отмеченное свойство, вообще говоря, не имеет места.

Напомним формулировки классических условий (и определения коэффициентов) перемешивания. Обозначим $\mathfrak{M}_{j}^{k}$, где $j \leqslant k, \sigma$-алгебру событий, порожденную случайными величинами $X_{j}, \ldots, X_{k}$.

О п р е д е л е н и е 3 . Последовательность $X_{1}, X_{2}, \ldots$ удовлетворяет условию $\alpha$-перемешивания (или сильного перемешивания), если при $i \rightarrow \infty$

$$
\alpha(i):=\sup _{k \geqslant 1} \sup _{A \in \mathfrak{M}_{1}^{k}, B \in \mathfrak{M}_{k+i}^{\infty}}|\mathbf{P}(A B)-\mathbf{P}(A) \mathbf{P}(B)| \rightarrow 0 .
$$

О п р е д е л е н и е 4 . Последовательность $X_{1}, X_{2}, \ldots$ удовлетворяет условию $\varphi$-перемешивания (или равномерно сильного перемешивания), если при $i \rightarrow \infty$

$$
\varphi(i):=\sup _{k \geqslant 1} \sup _{A \in \mathfrak{M}_{1}^{k}, B \in \mathfrak{M}_{k+i}^{\infty}, \mathbf{P}(A)>0} \frac{|\mathbf{P}(A B)-\mathbf{P}(A) \mathbf{P}(B)|}{\mathbf{P}(A)} \rightarrow 0 .
$$

О п р е д е л е н и е 5 . Последовательность $X_{1}, X_{2}, \ldots$ удовлетворяет условию $\psi$-перемешивания, если при $i \rightarrow \infty$

$$
\psi(i):=\sup _{k \geqslant 1} \sup _{A \in \mathfrak{M}_{1}^{k}, B \in \mathfrak{M}_{k+i}^{\infty}, \mathbf{P}(A) \mathbf{P}(B)>0} \frac{|\mathbf{P}(A B)-\mathbf{P}(A) \mathbf{P}(B)|}{\mathbf{P}(A) \mathbf{P}(B)} \rightarrow 0 .
$$

При этом предполагается, что $\psi(1)<\infty$.

Отметим, что последовательность $m$-зависимых случайных величин удовлетворяет условиям $\alpha$ - и $\varphi$-перемешивания, но может не удовлетворять условию $\psi$-перемешивания.

В работе [12] для независимых $\left\{X_{i}\right\}$ при условии $f\left(t_{1}, \ldots, t_{d}\right) \in$ $L_{2}\left(\mathfrak{X}^{d}, F^{d}\right)$ доказано, что

$$
U_{n} \stackrel{d}{\longrightarrow} \sum_{i_{1}, \ldots, i_{d}=1}^{\infty} f_{i_{1} \ldots i_{d}} \prod_{j=1}^{\infty} H_{\nu_{j}\left(i_{1}, \ldots, i_{d}\right)}\left(\tau_{j}\right)
$$

где $\left\{\tau_{i}\right\}$ - последовательность независимых случайных величин со стандартным нормальным распределением, $\nu_{j}\left(i_{1}, \ldots, i_{d}\right)$ - число индексов среди $i_{1}, \ldots, i_{d}$, равных натуральному $j$, а $H_{k}(x)$ - полиномы Эрмита, определенные формулой

$$
H_{k}(x)=(-1)^{k} e^{x^{2} / 2} \frac{d^{k}}{d x^{k}}\left(e^{-x^{2} / 2}\right)
$$

или с помощью рекуррентного соотношения

$$
H_{n+1}=x H_{n}(x)-n H_{n-1}(x), \quad H_{0}(x) \equiv 1, \quad H_{1}(x)=x .
$$


Трудность перехода к случаю стационарно связанных наблюдений заключается в том, что даже сходимость ряда почти наверное относительно распределения $F^{d}$ и наличие $\varphi$-перемешивания наблюдений не гарантируют даже слабую сходимость частичных сумм упомянутого выше ряда к ядру при подстановке вместо неслучайных аргументов зависимых наблюдений. В работе [8] (см. также [5]), где исследовался слабый предел $U$-статистики второго порядка при $n \rightarrow \infty$, построенной по наблюдениям с условием $\varphi$-перемешивания, этот момент был упущен. Там утверждается, что при $d=2$ в случае стационарно связанных наблюдений с $\varphi$-перемешиванием при условии сходимости рядов $\sum\left|\lambda_{k}\right|$ и $\sum \varphi^{1 / 2}(k)$ выполнено следуюшее предельное соотношение:

$$
U_{n} \stackrel{d}{\longrightarrow} \sum_{k=1}^{\infty} \lambda_{k}\left(\tau_{k}^{2}-1\right)
$$

где $\left\{\lambda_{k}\right\}$ - собственные числа интегрального оператора с симметричным ядром $f\left(t_{1}, t_{2}\right)$ из $(5)$, а $\left\{\tau_{k}\right\}$ - гауссовская последовательность центрированных случайных величин с ковариациями

$$
\mathbf{E} \tau_{k} \tau_{l}=\mathbf{E} e_{k}\left(X_{1}\right) e_{l}\left(X_{1}\right)+\sum_{j=1}^{\infty}\left[\mathbf{E} e_{k}\left(X_{1}\right) e_{l}\left(X_{j+1}\right)+\mathbf{E} e_{l}\left(X_{1}\right) e_{k}\left(X_{j+1}\right)\right]
$$

где $\left\{e_{k}(t)\right\}$ - ортонормированный базис из собственных функций упомянутого интегрального оператора.

В [2, предложение 2] был построен пример, в котором при выполнении всех условий из условий [8] ряд в правой части (5) при подстановке вместо неслучайных аргументов пары зависимых наблюдений из исходной стационарной последовательности не совпадает с положительной вероятностью с соответствующим значением ядра и, более того, слабый предел рассматриваемой $U$-статистики не существует. Вкратце напомним эту конструкцию.

Пусть $\left\{Y_{i} ; i \geqslant 1\right\}$ - последовательность независимых случайных величин, распределенных равномерно на отрезке $[-1,1]$, а $\left\{\xi_{i} ; i \geqslant 1\right\}-$ последовательность независимых между собой и от последовательности $\left\{Y_{i}\right\}$ бернуллиевских случайных величин с вероятностью успеха $1 / 2$. Положим $X_{i}=Y_{i+\xi_{i}}$. Случайные величины $X_{i}$ образуют стационарную последовательность 1-зависимых величин. Очевидно, что $X_{i}$ также равномерно распределены на $[-1,1]$. При этом для соответствующих независимых наблюдений $X_{1}^{*}$ и $X_{2}^{*}$ мы имеем $\mathbf{P}\left(X_{1}^{*}=X_{2}^{*}\right)=0$, а для оригинальных

$$
\mathbf{P}\left(X_{1}=X_{2}\right)=\mathbf{P}\left(\xi_{1}=1\right) \mathbf{P}\left(\xi_{2}=0\right)=\frac{1}{4} .
$$

Отметим, что в рассматриваемом примере ни базисные функции, ни коэффициенты разложения в (5) не зависят от значения ядра $f$ на диаго- 
нали ввиду неатомарности маргинального распределения (см. характеристическое уравнение для определения спектральных характеристик). Далее, в [2] показано, что в этом случае слабый предел $U$-статистики будет равен

$$
\frac{\mathbf{E} f\left(Y_{1}, Y_{1}\right)}{2}+\sum_{k \geqslant 1} \lambda_{k}\left(\tau_{k}^{2}-\frac{3}{2}\right)
$$

где $\lambda_{k}$ и $\tau_{k}$ те же, что в (7). Поскольку в условиях работы [8] величина $\mathbf{E} f\left(Y_{1}, Y_{1}\right)$ может быть любым числом или даже не существовать, то приведенный слабый предел в общем случае не совпадает с (7).

В работах [1], [2], [7] были получены результаты, аналогичные [12], для стационарно связанных наблюдений. В силу вышесказанного для применения тех же методов в случае зависимых наблюдений потребовались дополнительные ограничения. Например, в [2] были введены следующие условия.

(AC) Для любого набора попарно различньх индексов $\left(j_{1}, \ldots, j_{d}\right)$ распределение вектора $\left(X_{j_{1}}, \ldots, X_{j_{d}}\right)$ абсолютно непрерьвно относительно распределения вектора $\left(X_{1}^{*}, \ldots, X_{d}^{*}\right)$.

(R) Ядро $f\left(t_{1}, \ldots, t_{d}\right)$ непрерьввно всюду на $\mathfrak{X}^{d}$ (по каждой переменной), и все базиснье элементы $e_{k}(t)$ в (4) непрерьвны и равномерно по $t$ и $k$ ограниченьл.

Отметим, что каждое из этих двух условий обеспечивает совпадение с вероятностью 1 ядра и его разложения в упомянутый ортогональный ряд (при условии абсолютной суммируемости его коэффициентов) при подстановке вместо аргументов пары зависимых наблюдений из исходной стационарной последовательности.

В работе [2] доказано, что в случае $\alpha$ - и $\varphi$-перемешивания при выполнении условия (AC), условия абсолютной суммируемости коэффициентов $f_{i_{1} \ldots i_{d}}$ и при некоторых ограничениях на моменты базисных элементов, а также на скорость убывания коэффициентов перемешивания верно следующее предельное соотношение:

$$
U_{n} \stackrel{d}{\longrightarrow} \sum_{i_{1}, \ldots, i_{d}=1}^{\infty} f_{i_{1} \ldots i_{d}} \prod_{j=1}^{\infty} H_{\nu_{j}\left(i_{1}, \ldots, i_{d}\right)}\left(\tau_{j}\right)
$$

Заменив условие $(\mathrm{AC})$ на $(\mathrm{R})$, получаем предельную теорему для $V$ статистик (подробнее см. [2]):

$$
V_{n} \stackrel{d}{\rightarrow} \sum_{i_{1}, \ldots, i_{d}=1}^{\infty} f_{i_{1} \ldots i_{d}} \tau_{i_{1}} \cdots \tau_{i_{d}}
$$

В обоих случаях $\left\{\tau_{i}\right\}$ - центрированная гауссовская последовательность 
с ковариациями

$$
\mathbf{E} \tau_{k} \tau_{l}=\delta_{k l}+\sum_{j=1}^{\infty}\left[\mathbf{E} e_{k}\left(X_{1}\right) e_{l}\left(X_{j+1}\right)+\mathbf{E} e_{l}\left(X_{1}\right) e_{k}\left(X_{j+1}\right)\right] .
$$

Требованию (АС) удовлетворяют, к примеру, последовательности с $\psi$-перемешиванием (для которых предельный закон сформулирован в [1]). Но легко проверить, что приведенный выше пример последовательности 1-зависимых случайных величин этому требованию не удовлетворяет.

2. Основные результаты. Цель настоящей работы - получить предельные теоремы для $U$ - и $V$-статистик, построенных по выборкам из последовательности $m$-зависимых случайных величин, без ограничительных условий на совместные распределения элементов исходной последовательности или условий регулярности типа условий $(\mathrm{AC})$ и $(\mathrm{R})$.

Предлагаемый в настоящей работе подход, как и в упомянутых работах [1] и [2], также основан на разложении ядра в кратный ряд, но в данном случае он будет несколько отличаться от (4).

В силу определения $m$-зависимости каждый случайный вектор аргументов $\left\{X_{j_{1}}, \ldots, X_{j_{d}}\right\}$ соответствующего ядра (индексы которых в случае $V$-статистик могут иметь различную кратность) можно разбить на независимые между собой подвекторы (блоки), количество которых может принимать значения от 1 до $d$. Назовем блок неделимым, если после упорядочения по возрастанию индексов (чего не нужно делать для $U$-статистик), определяющих данный блок, расстояние между любыми соседними индексами не будет превышать $m$. Дадим более формальное определение. Пусть $\bar{k}=\left\{j_{k_{1}}, \ldots, j_{k_{l}}\right\} \subset\left\{j_{1}, \ldots, j_{d}\right\}$. Будем считать, что элементы $\left\{X_{j_{k_{1}}}, \ldots, X_{j_{k_{l}}}\right\}$ образуют неделимый блок, если для каждого $j \in \bar{k}$ выполнено

$$
\min _{j^{\prime} \in \bar{k} \backslash\{j\}}\left|j-j^{\prime}\right| \leqslant m \quad \text { и } \quad \min _{j^{\prime} \in\left\{j_{1}, \ldots, j_{d}\right\} \backslash \bar{k}}\left|j-j^{\prime}\right|>m .
$$

Отсюда следует, что все блоки (подвекторы) внутри конкретного набора $\left\{X_{j_{1}}, \ldots, X_{j_{d}}\right\}$ будут независимыми в совокупности.

В дальнейшем, говоря о неделимых блоках $\left\{X_{j_{k_{1}}}, \ldots, X_{j_{k_{l}}}\right\}$, мы будем иногда оперировать «неделимыми блоками» соответствующих индексов $\left\{j_{k_{1}}, \ldots, j_{k_{l}}\right\}$.

Заметим, что распределение вектора наблюдений $\left\{X_{j_{1}}, \ldots, X_{j_{d}}\right\}$ однозначно определяется распределениями блоков, на которые он разбивается, причем перестановка индексов внутри отдельного блока, вообще говоря, может изменить его распределение. Следовательно, всевозможных различных распределений $\left\{X_{j_{1}}, \ldots, X_{j_{d}}\right\}$ будет конечное число, зависящее только от $d$ и $m$. 
Далее, разобьем множество $d$-мерных мультииндексов

$$
\left\{\left(j_{1}, \ldots, j_{d}\right): j_{r}=1, \ldots, n ; r=1, \ldots, d\right\}
$$

на непересекающиеся классы эквивалентности $\left\{K_{l}\right\}$, число которых зависит лишь от параметров $d$ и $m$. Мультииндексы каждого такого класса содержат фиксированное число блоков и получаются один из другого некоторыми параллельньлми сдвигами указанных блоков (т.е. прибавлением ко всем координатам отдельного блока одного и того же целого числа, но своего для каждого блока) при одном лишь условии, что расстояние Хаусдорфа между каждой парой указанных блоков должно превосходить число $m$ (чтобы число сдвигаемых блоков было постоянным).

Пусть мультииндексы некоторого класса $K_{l}$ разбиваются на $s$ неделимых блоков. Если $s>d / 2$, потребуем, чтобы

$$
\mathbf{E} f^{2}\left(X_{j_{1}}, \ldots, X_{j_{d}}\right)<\infty .
$$

Если же $s \leqslant d / 2$, будем требовать лишь конечность первого момента ядра:

$$
\mathbf{E}\left|f\left(X_{j_{1}}, \ldots, X_{j_{d}}\right)\right|<\infty .
$$

Стоит сразу заметить, что общее число элементов $K_{l}$ не превышает $n^{s}$ и эквивалентно $n^{s}$ при $n \rightarrow \infty$.

Предложение 1. Пусть для класса $K_{l}$ с иислом блоков $s=d / 2$ выполнено условие (13). Тогда

$$
n^{-d / 2} \sum_{K_{l}} f\left(X_{j_{1}}, \ldots, X_{j_{d}}\right) \stackrel{\mathbf{P}}{\longrightarrow} \mathbf{E} f\left(X_{j_{1}^{0}}, \ldots, X_{j_{d}^{0}}\right)
$$

при $n \rightarrow \infty$, где $\left(j_{1}^{0}, \ldots, j_{d}^{0}\right)$ - любой представитель класса $K_{l}$.

Д о к а з а т е л ь с т в о. Фактически мы имеем дело со статистикой вида

$$
S_{n}=n^{-s} \sum_{\left(\bar{k}_{1}, \ldots, \bar{k}_{s}\right) \in K_{l}} f_{0}\left(\bar{X}_{\bar{k}_{1}}, \ldots, \bar{X}_{\bar{k}_{s}}\right),
$$

где функция $f_{0}$ есть суперпозиция исходного ядра $f$ и оператора, обратного к оператору перестановки аргументов по возрастанию их индексов (для симметричных относительно перестановки аргументов ядер имеем тождество $\left.f_{0} \equiv f\right)$; каждая из последовательностей $\left\{\bar{X}_{\bar{k}_{i}}\right\}(i=1, \ldots, s)$ состоит из $m_{k}$-зависимых случайных векторов (блоков), вообще говоря, различной размерности с одним и тем же маргинальным распределением в каждой последовательности. При этом для любого набора блочных мультииндексов $\left(\bar{k}_{1}, \ldots, \bar{k}_{s}\right) \in K_{l}$ набор $\left(\bar{X}_{\bar{k}_{1}}, \ldots, \bar{X}_{\bar{k}_{s}}\right)$ состоит из независимых блоков. Кроме того, если расстояние Хаусдорфа между наборами блочных мультииндексов $\left(\bar{k}_{1}, \ldots, \bar{k}_{s}\right)$ и $\left(\bar{r}_{1}, \ldots, \bar{r}_{s}\right)$ превосходит число $m$, 
то соответствующие векторные наборы $\left(\bar{X}_{\bar{k}_{1}}, \ldots, \bar{X}_{\bar{k}_{s}}\right)$ и $\left(\bar{X}_{\bar{r}_{1}}, \ldots, \bar{X}_{\bar{r}_{s}}\right)$ также независимы.

Для случайной величины $X$ обозначим $\|X\|_{p}:=\left(\mathbf{E}|X|^{p}\right)^{1 / p}, p \geqslant 1$.

Пусть $N$ - произвольное положительное число. Введем в рассмотрение срезку и ее хвост для ядра $f_{0}$ :

$$
\begin{aligned}
& f_{0}^{(N)}\left(t_{1}, \ldots, t_{s}\right):=f_{0}\left(t_{1}, \ldots, t_{s}\right) I\left(\left|f_{0}\left(t_{1}, \ldots, t_{s}\right)\right| \leqslant N\right), \\
& \bar{f}_{0}^{(N)}\left(t_{1}, \ldots, t_{s}\right):=f_{0}\left(t_{1}, \ldots, t_{s}\right)-f_{0}^{(N)}\left(t_{1}, \ldots, t_{s}\right) .
\end{aligned}
$$

Тогда имеем

$$
\left\|S_{n}-\mathbf{E} S_{n}\right\|_{1} \leqslant\left\|S_{n}^{(N)}-\mathbf{E} S_{n}^{(N)}\right\|_{1}+\left\|\bar{S}_{n}^{(N)}-\mathbf{E} \bar{S}_{n}^{(N)}\right\|_{1},
$$

где $S_{n}^{(N)}$ и $\bar{S}_{n}^{(N)}$ строятся по формуле $(15)$ для срезки и хвоста срезки ядра соответственно. Очевидно, что $\mathbf{E} S_{n}^{(N)} \rightarrow \mathbf{E} S_{n}$ и

$$
\sup _{n}\left\|\bar{S}_{n}^{(N)}-\mathbf{E} \bar{S}_{n}^{(N)}\right\|_{1} \leqslant 2\left\|\bar{f}_{0}^{(N)}\left(\bar{X}_{\bar{j}_{1}^{0}}, \ldots, \bar{X}_{\bar{j}_{s}^{0}}\right)\right\|_{1} \rightarrow 0
$$

при $N \rightarrow \infty$. Остается доказать, что для любого фиксированного $N$ при $n \rightarrow \infty$

$$
\left\|S_{n}^{(N)}-\mathbf{E} S_{n}^{(N)}\right\|_{1} \rightarrow 0
$$

В силу неравенства Коши-Буняковского нам достаточно установить предыдушее соотношения для нормы $\|\cdot\|_{2}$, которая для срезанной статистики, очевидно, существует. Обозначим через $\tilde{f}_{0}^{(N)}$ центрированную срезку ядра. Тогда легко видеть, что

$$
\begin{aligned}
\| S_{n}^{(N)} & -\mathbf{E} S_{n}^{(N)} \|_{2}^{2} \\
& =\frac{1}{n^{2 s}} \sum_{\left(\bar{k}_{1}, \ldots, \bar{k}_{s}\right) \in K_{l}} \sum_{\left(\bar{r}_{1}, \ldots, \bar{r}_{s}\right) \in K_{l}} \mathbf{E} \tilde{f}_{0}^{(N)}\left(\bar{X}_{\bar{k}_{1}}, \ldots, \bar{X}_{\bar{k}_{s}}\right) \tilde{f}_{0}^{(N)}\left(\bar{X}_{\bar{r}_{1}}, \ldots, \bar{X}_{\bar{r}_{s}}\right) \\
& =O\left(\frac{1}{n}\right)
\end{aligned}
$$

поскольку смешанный момент под знаком кратной суммы может быть отличен от нуля, только если расстояние Хаусдорфа между наборами индексов $\left(\bar{k}_{1}, \ldots, \bar{k}_{s}\right)$ и $\left(\bar{r}_{1}, \ldots, \bar{r}_{s}\right)$ не превосходит $m$, что означает, что число «степеней свободы» в пространстве индексов не превосходит $2 s-1$, т.е. число ненулевых слагаемых (мажорируемых одним числом) в указанной кратной сумме имеет порядок $O\left(n^{2 s-1}\right)$. Предложение доказано.

Если же $s<d / 2$, то из вышеприведенных выкладок следует, что

$$
n^{-d / 2} \sum_{K^{l}} f\left(X_{j_{1}}, \ldots, X_{j_{d}}\right) \stackrel{\mathbf{P}}{\longrightarrow} 0
$$

при $n \rightarrow \infty$. 
Таким образом, классы, в которых $s<d / 2$, не играют никакой роли для описания слабого предела рассматриваемой статистики. Следовательно, в рассуждениях мы можем добавлять и отнимать слагаемые, составленные из них для удобства выкладок, не изменяя предельного значения.

Остановимся на ситуации, когда $d>2$ четно и $s=d / 2$. Если длина некоторого блока в классе больше двух, значит, в этом классе есть другой блок, длина которого равна единице. А тогда в силу независимости блоков и вырожденности ядра

$$
\mathbf{E} f\left(X_{j_{1}}, \ldots, X_{j_{d}}\right)=0,
$$

следовательно, ненулевой предел при $s=d / 2$ дают только классы, в которых векторы $\left(X_{j_{1}}, \ldots, X_{j_{d}}\right)$ состоят лишь из двухэлементных неделимых блоков.

Теперь рассмотрим случай, когда возможно разбиение $\left\{X_{j_{1}}, \ldots, X_{j_{d}}\right\}$ на $s>d / 2$ неделимых блоков. Наряду с неделимыми блоками $\bar{k}_{r}$ будем оперировать классами эквивалентности блоков $T_{r}$. Векторы $T_{r}$ получаются друг из друга параллельным переносом. Количество элементов в неделимых блоках класса $T_{r}$ будем обозначать \# $\left(T_{r}\right)$, минимальную «координату» блока $-\min \left(\bar{k}_{r}\right)$, расстояние Хаусдорфа между двумя блоками $-d\left(\bar{k}_{u}, \bar{k}_{r}\right)$.

Из условия (12) вытекает, что $f_{0}\left(u_{1}, \ldots, u_{s}\right) \in L_{2}\left(\mathfrak{X}^{\#\left(T_{1}\right)} \times \cdots \times\right.$ $\left.\mathfrak{X}^{\#\left(T_{s}\right)}, F_{T_{1}} \times \cdots \times F_{T_{s}}\right)$, где $u_{r} \in \mathfrak{X}^{\#\left(T_{r}\right)}, F_{T_{r}}$ - распределение векторов $T_{r}$.

Таким образом, ситуация сводится к случаю независимых аргументов, описанному в [12] (см. также [5], [2]). В каждом пространстве $L_{2}\left(\mathfrak{X}^{\#\left(T_{r}\right)}, F_{T_{r}}\right)$ можно также построить ортонормированный базис $\left\{e_{l}^{(r)}\left(u_{r}\right)\right\}_{l \geqslant 0}$, такой, что $e_{0}^{(r)}\left(u_{r}\right) \equiv 1$. Из ортогональности следует, что $\mathbf{E} e_{l}^{(r)}\left(\bar{X}_{\bar{k}_{r}}\right)=0$ для $l \geqslant 1$.

Далее, семейство функций

$$
\left\{e_{l_{1}}^{(1)}\left(u_{1}\right) \cdots e_{l_{s}}^{(s)}\left(u_{s}\right)\right\}_{l_{1}, \ldots, l_{s}=0,1, \ldots}
$$

будет ортонормированным базисом гильбертова пространства

$$
L_{2}\left(\mathfrak{X}^{\#\left(T_{1}\right)} \times \cdots \times \mathfrak{X}^{\#\left(T_{s}\right)}, F_{T_{1}} \times \cdots \times F_{T_{s}}\right) .
$$

Тогда $f_{0}\left(u_{1}, \ldots, u_{s}\right)$ можно представить в виде ряда

$$
f_{0}\left(u_{1}, \ldots, u_{s}\right)=\sum_{l_{1}, \ldots, l_{s}=0}^{\infty} f_{l_{1} \ldots l_{s}} e_{l_{1}}^{(1)}\left(u_{1}\right) \cdots e_{l_{s}}^{(s)}\left(u_{s}\right),
$$

частичные суммы которого сходятся в норме упомянутого пространства. Соответственно, подставив вместо переменных $\left\{u_{1}, \ldots, u_{s}\right\}$ случайные величины $\left\{\bar{X}_{\bar{k}_{1}}, \ldots, \bar{X}_{\bar{k}_{s}}\right\}$, принадлежащие интересующему нас классу, 
получим ряд, частичные суммы которого сходятся в среднеквадратичном к ядру $f\left(X_{j_{1}}, \ldots, X_{j_{d}}\right)$.

Очевидно, что в случаях \# $\left(T_{r}\right)=1$ базис $\left\{e_{l}^{(r)}(t)\right\}_{l \geqslant 0}$ является базисом пространства $L_{2}(\mathfrak{X}, F)$. Без ограничения общности можно считать его совпадающим с ранее введенным базисом $\left\{e_{l}(t)\right\}_{l \geqslant 0}$.

Для формулировки основных результатов введем некоторые понятия и обозначения. Пусть $\left\{\tau_{i}\right\}$ - гауссовская центрированная последовательность с ковариациями

$M_{k l}=\mathbf{E} \tau_{k} \tau_{l}=\mathbf{E} e_{k}\left(X_{1}\right) e_{l}\left(X_{1}\right)+\sum_{j=1}^{m}\left[\mathbf{E} e_{k}\left(X_{1}\right) e_{l}\left(X_{j+1}\right)+\mathbf{E} e_{l}\left(X_{1}\right) e_{k}\left(X_{j+1}\right)\right]$

Нетрудно видеть, что

$$
M_{k l}=\delta_{k l}+\sum_{j=1}^{m}\left[\mathbf{E} e_{k}\left(X_{1}\right) e_{l}\left(X_{j+1}\right)+\mathbf{E} e_{l}\left(X_{1}\right) e_{k}\left(X_{j+1}\right)\right]
$$

где $\delta_{k l}$ - символ Кронекера. Очевидно, что в случае $m$-зависимости выражение (18) совпадает с (11).

При этом в (18) допускается вырожденность в нуле тех или иных $\tau_{i}$ при $M_{i i}=0$.

Пусть $I\left(l_{1}, \ldots, l_{s}, q\right)$, где $q \leqslant s / 2$, - множество выборок (без возвращения и фиксации порядка извлечения) объема $q$, элементами которых являются пары чисел из множества индексов $\left\{l_{1}, \ldots, l_{s}\right\}$, которые предполагаются попарно различимыми. Количество всевозможных таких выборок совпадает с числом всевозможных вариантов фиксации в множестве натуральных чисел $\{1, \ldots, s\}$ ровно $q$ пар без общих элементов:

$$
\frac{s !}{2^{q}(s-2 q) ! q !}
$$

Введем обозначения:

$$
\begin{aligned}
p & =\left\{\left\{l_{i_{1}}, l_{i_{2}}\right\}, \ldots,\left\{l_{i_{2 q-1}}, l_{i_{2 q}}\right\}\right\} \in I\left(l_{1}, \ldots, l_{s}, q\right) ; \\
M_{p} & =M_{l_{i_{1}} l_{i_{2}}} \cdots M_{l_{i_{2 q-1}}, l_{i_{2 q}}} \\
p^{c} & =\left\{l_{1}, \ldots, l_{s}\right\} \backslash\left(\left\{l_{i_{1}}, l_{i_{2}}\right\} \cup \cdots \cup\left\{l_{i_{2 q-1}}, l_{i_{2 q}}\right\}\right) .
\end{aligned}
$$

Зафиксируем некоторый класс эквивалентности $K$ векторов, разбивающихся на $s \geqslant[d / 2]+1$ неделимых блоков, из которых $s_{1}$ одноэлементных, а остальные двухэлементные. Обозначим множество таких классов $\mathfrak{K}^{\left(s_{1}\right)}$. Отдельно выделим те классы из $\mathfrak{K}^{\left(s_{1}\right)}$, в которых все двухэлементные блоки образованы двумя различными координатами. Обозначим множество таких классов $\mathfrak{K}_{1}^{\left(s_{1}\right)}$. Пусть $\left\{X_{j_{1}}, \ldots, X_{j_{d}}\right\} \in K$ и одноэлементные блоки соответствуют в этом векторе координатам $j_{k_{1}}, \ldots, j_{k_{s_{1}}}$, 
где $1 \leqslant k_{1}<\cdots<k_{s_{1}} \leqslant d$. Тогда выделим коэффициенты разложения (17) при таких базисных функциях $e_{l_{1}}^{(1)} \cdots e_{l_{s}}^{(s)}$, где одноэлементным блокам соответствуют некоторые $e_{i_{1}}, \ldots, e_{i_{s_{1}}}$, а двухэлементным - нулевой элемент базиса, тождественно равный 1 :

$$
f_{i_{1} \ldots i_{s_{1}}}^{(K)}=\mathbf{E} f\left(X_{j_{1}}, \ldots, X_{j_{d}}\right) e_{i_{1}}\left(X_{j_{k_{1}}}\right) \cdots e_{i_{s_{1}}}\left(X_{j_{k_{s_{1}}}}\right) .
$$

Основные результаты работы содержатся в следующих двух теоремах.

Теорема 1. Пусть каноническое ядро $f\left(t_{1}, \ldots, t_{d}\right)$ и последовательность $m$-зависимых случайных величин $\left\{X_{i}\right\}$ таковь, что для всех мультииндексов $\left\{j_{1}, \ldots, j_{d}\right\}$, разбивающихся на более чем $[d / 2]$ неделимых блоков, выполнено (12), для остальных выполнено (13). Тогда

$$
\begin{aligned}
V_{n} \stackrel{d}{\longrightarrow} \sum_{k=0}^{[(d-1) / 2]} \sum_{i_{1}, \ldots, i_{d-2 k}=1}^{\infty} \sum_{K \in \mathfrak{K}^{(d-2 k)}} f_{i_{1} \ldots i_{d-2 k}}^{(K)} \sum_{q=0}^{[d / 2-k]}(-1)^{q} \\
\times \sum_{p \in I\left(i_{1}, \ldots, i_{d-2 k}, q\right)} M_{p} \prod_{i \in p^{c}} \tau_{i}+C,
\end{aligned}
$$

где ряд в правой части сходится в среднеквадратичном, $\left\{\tau_{i}\right\}-$ гауссовская центрированная последовательность с ковариациями (18); $C=0$ в случае нечетного $d$, в противном случае

$$
C=\sum_{K^{\prime} \in \mathfrak{K}(0)} \mathbf{E} f\left(X_{j_{1}^{\prime}}, \ldots, X_{j_{d}^{\prime}}\right) .
$$

Здесь $\left\{X_{j_{1}^{\prime}}, \ldots, X_{j_{d}^{\prime}}\right\}-$ некоторый вектор из класса $K^{\prime}$.

Теорема 2. Пусть условия теоремы 1 выполнены для мультииндексов $\left\{j_{1}, \ldots, j_{d}\right\}$ из области суммирования кратной суммь в (3). Тогда

$$
\begin{aligned}
U_{n} \stackrel{d}{\longrightarrow} & \sum_{k=0}^{[(d-1) / 2]} \sum_{i_{1}, \ldots, i_{d-2 k}=1}^{\infty} \sum_{K \in \mathfrak{K}_{1}^{(d-2 k)}} f_{i_{1} \ldots i_{d-2 k}}^{(K)} \sum_{q=0}^{[d / 2-k]}(-1)^{q} \\
& \times \sum_{p \in I\left(i_{1}, \ldots, i_{d-2 k}, q\right)} M_{p} \prod_{i \in p^{c}} \tau_{i}+C
\end{aligned}
$$

где ряд в правой части сходится в среднеквадратичном, $\tau_{i}$ определеньи выце, $C=0$ в случае нечетного $d$, в противном случае

$$
C=\sum_{K^{\prime} \in \mathfrak{K}_{1}^{(0)}} \mathbf{E} f\left(X_{j_{1}^{\prime}}, \ldots, X_{j_{d}^{\prime}}\right) .
$$

Здесь $\left\{X_{j_{1}^{\prime}}, \ldots, X_{j_{d}^{\prime}}\right\}-$ некоторый вектор из класса $K^{\prime}$.

3 а м е ч а н и е 1 . Сумму $\sum_{K \in \mathfrak{K}^{(d-2 k)}}$ можно расписать следующим образом:

$$
\sum_{K \in \mathfrak{K}^{(d-2 k)}}=\sum_{1 \leqslant k_{d-2} k+1<\cdots<k_{d} \leqslant d} \sum_{p_{1} \in I\left(k_{d-2 k+1}, \ldots, k_{d}, k\right)} \sum_{-m \leqslant m_{1}, \ldots, m_{k} \leqslant m},
$$


где $k_{d-2 k+1}, \ldots, k_{d}$ - номера координат, входящих в состав двухэлементных блоков, $p_{1}$ - разбиение их на $k$ пар, $m_{i}$ - разность между большей и меньшей координатами $i$-го двухэлементного блока (эти блоки можно упорядочить, скажем, по возрастанию меньшей координаты). образом:

Сумма $\sum_{K^{\prime} \in \mathfrak{K}(0)}$, как частный случай $(23)$, записывается следующим

$$
\sum_{K^{\prime} \in \mathfrak{K}^{(0)}}=\sum_{p^{\prime} \in I(1, \ldots, d, d / 2)} \sum_{-m \leqslant m_{1}, \ldots, m_{d / 2} \leqslant m} .
$$

Соответственно, сумма $\sum_{K \in \mathfrak{K}_{1}^{(d-2 k)}}$ расписывается как

$$
\sum_{K \in \mathfrak{K}_{1}^{(d-2 k)}}=\sum_{1 \leqslant k_{d-2} k+1<\cdots<k_{d} \leqslant d} \sum_{p \in I\left(k_{d-2 k+1}, \ldots, k_{d}, k\right)} \sum_{1 \leqslant\left|m_{1}\right|, \ldots,\left|m_{k}\right| \leqslant m}
$$

a сумма $\sum_{K^{\prime} \in \mathfrak{K}_{1}^{(0)}}-$ каK

$$
\sum_{K^{\prime} \in \mathfrak{K}_{1}^{(0)}}=\sum_{p^{\prime} \in I(1, \ldots, d, d / 2)} \sum_{1 \leqslant\left|m_{1}\right|, \ldots,\left|m_{d / 2}\right| \leqslant m} .
$$

3 а м е ч а н и е 2. В заключение работы будет показано, что при выполнении как условий теоремы 1, так и условий теоремы 2 из [2] правая часть (21) совпадает с правой частью (10). Аналогично, при выполнении как условий теоремы 2, так и условий теоремы 1 из [2] правая часть (22) совпадает с правой частью (9).

$\Pi$ р и м е р. При $d=2$ слабый предел $V$-статистики имеет вид

$$
\sum_{i, j=1}^{\infty} f_{i j}\left(\tau_{i} \tau_{j}-M_{i j}\right)+\sum_{k=-m}^{m} \mathbf{E} f\left(X_{m+1}, X_{k+m+1}\right)
$$

где

$$
f_{i j}=\mathbf{E} f\left(X_{1}, X_{m+2}\right) e_{i}\left(X_{1}\right) e_{j}\left(X_{m+2}\right) .
$$

Если же $d=3$, то слабый предел статистики Мизеса выглядит следующим образом:

$$
\begin{aligned}
& \sum_{i_{1}, i_{2}, i_{3}=1}^{\infty} f_{i_{1} i_{2} i_{3}}\left(\tau_{i_{1}} \tau_{i_{2}} \tau_{i_{3}}-M_{i_{1} i_{2}} \tau_{i_{3}}-M_{i_{1} i_{3}} \tau_{i_{2}}-M_{i_{2} i_{3}} \tau_{i_{1}}\right) \\
& \quad+\sum_{k=-m}^{m} \sum_{i=1}^{\infty}\left(f_{i}^{k, 1}+f_{i}^{k, 2}+f_{i}^{k, 3}\right) \tau_{i}
\end{aligned}
$$

где

$$
\begin{aligned}
f_{i_{1} i_{2} i_{3}} & =\mathbf{E} f\left(X_{1}, X_{m+2}, X_{2 m+3}\right) e_{i_{1}}\left(X_{1}\right) e_{i_{2}}\left(X_{m+2}\right) e_{i_{3}}\left(X_{2 m+3}\right), \\
f_{i}^{k, 1} & =\mathbf{E} f\left(X_{3 m+2}, X_{m+1}, X_{m+k+1}\right) e_{i}\left(X_{3 m+2}\right), \\
f_{i}^{k, 2} & =\mathbf{E} f\left(X_{m+1}, X_{3 m+2}, X_{m+k+1}\right) e_{i}\left(X_{3 m+2}\right), \\
f_{i}^{k, 3} & =\mathbf{E} f\left(X_{m+1}, X_{m+k+1}, X_{3 m+2}\right) e_{i}\left(X_{3 m+2}\right) .
\end{aligned}
$$




\section{3. Доказательство теорем 1,2 и замечания 2.}

Д о к а з а т е л ь с т в о т е о р е м ы 1. Для нахождения слабого предела $V$-статистики разобьем область суммирования в (2) на введенные выше классы, количество которых не зависит от $n$. Сначала найдем предельное распределение нормированной суммы по мультииндексам фиксированного класса.

Пусть векторы фиксированного класса разбиваются на $s>[d / 2]$ неделимых блоков. Для каждого $T_{i}$ зафиксируем произвольный элемент соответствующего базиса с номером $l_{i}$. Найдем слабый предел выражения

$$
n^{-d / 2} \sum_{*} e_{l_{1}}^{(1)}\left(\bar{X}_{\bar{k}_{1}}\right) \cdots e_{l_{s}}^{(s)}\left(\bar{X}_{\bar{k}_{s}}\right),
$$

где $\sum_{*}$ обозначает суммирование по всем векторам выделенного класса. В сумме (24) индексы каждого блока пробегают значения от 1 до $n$ (с точностью до длины блока), при этом расстояния между индексами различных блоков должны быть больше $m$. Добавляя и отнимая соответствующие слагаемые, можно представить (24) в виде линейной комбинации произведений величин вида

$$
S(T)=n^{-\#(T) / 2} \sum_{\min (\bar{k})=1}^{n} \widetilde{e}\left(\bar{X}_{\bar{k}}\right),
$$

где \# - считающая мера, $\bar{X}_{\bar{k}}$ - некоторый блок наблюдений (а вектор $\bar{k}$, соответственно, - индексов), либо совпадающий с одним из $\bar{k}_{i}$, либо «склеенный» из нескольких. Расстояния между соседними индексами в $\bar{k}$ не превышают $m$. Сумма длин блоков в каждом произведении равна $d$. Первым слагаемым в упомянутой линейной комбинации является $S\left(T_{1}\right) \cdots S\left(T_{s}\right)$, в остальных слагаемых количество блоковсомножителей будет уменьшаться, а их длина соответственно увеличиваться.

Видно, что (25) является суммой $k$-зависимых одинаково распределенных величин, где $k \leqslant d m$. Если $\#(T)=2$, то $S(T)$ будет сходиться по вероятности к константе по закону больших чисел для $k$-зависимых случайных величин, так как первый момент слагаемых в $S(T)$ будет, очевидно, конечен.

Покажем, что в случае $\#(T)>2$ величина $S(T)$ сходится к нулю по вероятности. Ее можно расписать в виде

$$
S(T)=S_{1}+\cdots+S_{k},
$$

где все $S_{i}$ являются суммами независимых одинаково распределенных величин и количество слагаемых в каждой $S_{i}$ эквивалентно $n / k$. Рассмотрим одну из таких сумм, скажем, $S_{1}$, и покажем, что она стремится 
к нулю по вероятности при \# $(T)>2$. Имеем

$$
S_{1}=n^{-\#(T) / 2} \sum_{\min (\bar{k})=i k+1,0 \leqslant i \leqslant[n / k]-1} \widetilde{e}\left(\bar{X}_{\bar{k}}\right) .
$$

Как уже было отмечено, блок $\bar{k}$ «склеен» из одного или нескольких блоков $\bar{k}_{r}$, т.е. $\widetilde{e}\left(\bar{X}_{\bar{k}}\right)$ является произведением нескольких $e_{l_{r}}^{(r)}\left(\bar{X}_{\bar{k}_{r}}\right)$, только блоки-компоненты уже не будут независимыми. Требуемая сходимость к нулю будет доказана, если показать, что для любого $r$

$$
\max \left|n^{-1 / 2} e_{l_{r}}^{(r)}\left(\bar{X}_{\bar{k}_{r}}\right)\right| \stackrel{\mathbf{P}}{\longrightarrow} 0,
$$

где максимум ищется по всем $[n / k]$ слагаемым $S_{1}$. Так как все эти слагаемые независимы, то последнее соотношение выполняется, если имеет место асимптотическое соотношение $\mathbf{P}\left(\left|e_{l_{r}}^{(r)}\left(\bar{X}_{\bar{k}_{r}}\right)\right|>\varepsilon n^{1 / 2}\right)=o(1 / n)$ для произвольного фиксированного $\varepsilon>0$ или, иначе говоря, $\mathbf{P}\left(\left|e_{l_{r}}^{(r)}\left(\bar{X}_{\bar{k}_{r}}\right)\right|>\right.$ $x)=o\left(1 / x^{2}\right)$ при $x \rightarrow \infty$. Так как по построению $e_{l_{r}}^{(r)}\left(\bar{X}_{\bar{k}_{r}}\right)$ имеет конечный второй момент, равный единице, требуемое свойство выполнено.

Значит, если изначально существует такой блок, что $\#\left(T_{r}\right) \geqslant 3$, то и вся сумма (24) сходится к нулю по вероятности. Будем теперь рассматривать лишь те классы, в которых каждый блок содержит не более двух элементов.

В [2, доказательство теоремы 1] показано, что последовательность

$$
\left\{n^{-1 / 2} \sum_{j=1}^{n} e_{1}\left(X_{j}\right), n^{-1 / 2} \sum_{j=1}^{n} e_{2}\left(X_{j}\right), \ldots\right\}
$$

слабо сходится при $n \rightarrow \infty$ к центрированной гауссовской последовательности $\left\{\tau_{i}\right\}$ с ковариационной матрицей (18).

Заметим, что в случае \# $\left(T_{r}\right)=1$ в силу вырожденности ядра в разложении (17) коэффициенты $f_{l_{1} \ldots l_{s}}$ равны нулю при $l_{r}=0$ (подробнее см. [5], [2, предложение 1]), т.е. есть смысл рассматривать лишь элементы базиса $e_{l}(t)$ для $l \geqslant 1$.

Далее, если $\widetilde{e}\left(\bar{X}_{\bar{k}}\right)$ совпадает с некоторым $e_{l}^{(r)}\left(\bar{X}_{\bar{k}_{r}}\right)$, где \# $\left(T_{r}\right)=2$, TO

$$
n^{-1} \sum_{i=1}^{n} e_{l}^{(r)}\left(\bar{X}_{\bar{k}_{r}}\right) \stackrel{\mathbf{P}}{\longrightarrow} \mathbf{E} e_{l}^{(r)}\left(\bar{X}_{\bar{k}_{r}}\right)
$$

причем, как было замечено ранее, $\mathbf{E} e_{l}^{(r)}\left(\bar{X}_{\bar{k}_{r}}\right)=0$ при $l>0$ и $\mathbf{E} e_{l}^{(r)}\left(\bar{X}_{\bar{k}_{r}}\right)=1$ при $l=0$. Значит, в случае двухэлементных блоков стоит рассматривать лишь нулевой элемент базиса $e_{0}^{(r)}\left(\bar{X}_{\bar{k}_{r}}\right) \equiv 1$.

Пусть в нашем классе имеется $s_{1}$ одноэлементных блоков и $\left(d-s_{1}\right) / 2$ двухэлементных, причем в выражении (24) одноэлементным блокам соответствуют элементы базиса, отличные от нулевого, а двухэлементным, напротив, лишь тождественная единица. 
Видно, что $S(T)$ либо сходится по вероятности к константе, либо совпадает с некоторым элементом последовательности (26). Так как (24) представляет собой конечную линейную комбинацию произведений различных $S(T)$, вышесказанное позволяет получить предел по распределению для выражения в (24), заменив в этой линейной комбинации все $S(T)$ на их слабые пределы. Найдем этот предел.

В силу того, что двухэлементным блокам соответствует лишь нулевой элемент базиса, от них зависит лишь количество слагаемых в (24) при фиксированных одноэлементных $T_{r}$. Нетрудно проверить, что это количество будет эквивалентно $n^{\left(d-s_{1}\right) / 2}$ при $n \rightarrow \infty$. Таким образом, учитывая конечность моментов слагаемых, в пределе сумма (24) зависит лишь от одноэлементных блоков и экивалентна величине

$$
n^{-s_{1} / 2} \sum_{\left|u_{i}-u_{j}\right|>m, 1 \leqslant i \neq j \leqslant s_{1}} e_{l_{1}^{\prime}}\left(X_{u_{1}}\right) \cdots e_{l_{s_{1}}^{\prime}}\left(X_{u_{s_{1}}}\right)=: g_{s_{1}}\left(l_{1}^{\prime}, \ldots, l_{s_{1}}^{\prime}\right) .
$$

Лемма 1. Справедлива следующая сходимость распределений:

$$
g_{s_{1}}\left(l_{1}, \ldots, l_{s_{1}}\right) \stackrel{d}{\rightarrow} \sum_{q=0}^{\left[s_{1} / 2\right]}(-1)^{q} \sum_{p \in I\left(l_{1}, \ldots, l_{s_{1}}, q\right)} M_{p} \prod_{i \in p^{c}} \tau_{i}
$$

где $\left\{\tau_{i}\right\}-$ гауссовская последовательность с ковариачионной матриией (18).

Д о к а з а т е ль с т в о. Из того факта, что если $\#(T) \geqslant 3$, то $S(T) \rightarrow 0$ по вероятности при $n \rightarrow \infty$, нетрудно вывести следующую рекуррентную формулу для $g_{s_{1}}\left(l_{1}, \ldots, l_{s_{1}}\right)$ :

$$
\begin{aligned}
g_{s_{1}} & \left(l_{1}, \ldots, l_{s_{1}}\right) \\
= & n^{-1 / 2} \sum_{j=1}^{n} e_{l_{1}}\left(X_{j}\right) g_{s_{1}-1}\left(l_{2}, \ldots, l_{s_{1}}\right)-\sum_{i=2}^{s_{1}} g_{s_{1}-2}\left(l_{2}, \ldots, l_{i-1}, l_{i+1}, \ldots, l_{s_{1}}\right) n^{-1} \\
& \times\left(\sum_{j=1}^{n} e_{l_{1}}\left(X_{j}\right) e_{l_{i}}\left(X_{j}\right)+\sum_{k=1}^{m} \sum_{j=1}^{n-k}\left(e_{l_{1}}\left(X_{j}\right) e_{l_{i}}\left(X_{j+k}\right)+e_{l_{1}}\left(X_{j+k}\right) e_{l_{i}}\left(X_{j}\right)\right)\right) \\
& +o_{\mathbf{P}}(1) .
\end{aligned}
$$

Устремляя $n$ к бесконечности, получим рекуррентную асимптотическую формулу для пределов:

$$
\begin{aligned}
\lim g_{s_{1}}\left(l_{1}, \ldots, l_{s_{1}}\right)= & \tau_{l_{1}} \lim g_{s_{1}-1}\left(l_{2}, \ldots, l_{s_{1}}\right) \\
& -\sum_{i=2}^{s_{1}} M_{l_{1} l_{i}} \lim g_{s_{1}-2}\left(l_{2}, \ldots, l_{i-1}, l_{i+1}, \ldots, l_{s_{1}}\right) .
\end{aligned}
$$

Докажем (27) индукцией по $s_{1}$. Легко видеть, что для $s_{1}=1$ и $s_{1}=2$ утверждение леммы выполнено (считаем $g_{0} \equiv 1$ ). Далее, пусть оно вы- 
полнено для всех $s_{2}<s_{1}$. Тогда

$$
\begin{aligned}
\lim g_{s_{1}}\left(l_{1}, \ldots, l_{s_{1}}\right)= & \tau_{l_{1}} \sum_{q=0}^{\left[\left(s_{1}-1\right) / 2\right]}(-1)^{q} \sum_{p \in I\left(l_{2}, \ldots, l_{s_{1}}, q\right)} M_{p} \prod_{i \in p^{c}} \tau_{i} \\
& -\sum_{i=2}^{s_{1}} M_{l_{1} l_{i}} \sum_{q=0}^{\left[\left(s_{1}-2\right) / 2\right]}(-1)^{q} \sum_{p \in I\left(l_{2}, \ldots, l_{i-1}, l_{i+1}, \ldots, l_{s_{1}}, q\right)} M_{p} \prod_{j \in p^{c}} \tau_{j} .
\end{aligned}
$$

Для $q \leqslant\left[\left(s_{1}-1\right) / 2\right]$ множество $I\left(l_{1}, \ldots, l_{s_{1}}, q\right)$ состоит из элементов $I\left(l_{2}, \ldots, l_{s_{1}}, q\right)$ и элементов вида $\left\{l_{1}, l_{i}\right\} \cup p$, где $p \in I\left(l_{2}, \ldots, l_{i-1}, l_{i+1}, \ldots, l_{s_{1}}\right.$, $q-1)$, а в случае четного $s_{1}$ для $q=s_{1} / 2$ - только из элементов второго типа. Учитывая это, получаем утверждение леммы.

3 а м е ч а н и е 3 . В случае $m=0$ (т.е. когда мы имеем дело с независимыми наблюдениями) $M_{i j}=\delta_{i j}$, величины $\tau_{i}$ являются независимыми стандартными нормальными величинами, а выражение в правой части (27) совпадает с $\prod_{j=1}^{\infty} H_{\nu_{j}\left(l_{1}, \ldots, l_{s_{1}}\right)}\left(\tau_{j}\right)$, определенным ранее в $(6)$.

Лемма 2. Пусть векторы фиксированного класса $K$ разбиваются на $s \geqslant[d / 2]+1$ неделимьх блоков, из которых $s_{1}$ одноэлементных. Тогдa

1) если в этом классе существует такое $T_{u}$, ито $\#\left(T_{u}\right) \geqslant 3$, то

$$
n^{-d / 2} \sum_{1 \leqslant j_{1}, \ldots, j_{d} \leqslant n:\left\{X_{j_{1}}, \ldots, X_{j_{d}}\right\} \in K} f\left(X_{j_{1}}, \ldots, X_{j_{d}}\right) \stackrel{d}{\rightarrow} 0 ;
$$

2) если длиньи всех блоков не превышают 2, то

$$
\begin{aligned}
& n^{-d / 2} \sum_{1 \leqslant j_{1}, \ldots, j_{d} \leqslant n:\left\{X_{j_{1}}, \ldots, X_{j_{d}}\right\} \in K} f\left(X_{j_{1}}, \ldots, X_{j_{d}}\right) \\
& \stackrel{d}{\longrightarrow} \sum_{i_{1}, \ldots, i_{s_{1}}=1}^{\infty} f_{i_{1} \ldots i_{s_{1}}}^{(K)} \sum_{q=0}^{\left[s_{1} / 2\right]}(-1)^{q} \sum_{p \in I\left(i_{1}, \ldots, i_{s_{1}}, q\right)} M_{p} \prod_{i \in p^{c}} \tau_{i},
\end{aligned}
$$

где ряд в правой части сходится в среднеквадратичном.

Д ок а з а т е л ь с т в о. В силу (17)

$$
\begin{aligned}
& n^{-d / 2} \sum_{1 \leqslant j_{1}, \ldots, j_{d} \leqslant n:\left\{X_{j_{1}}, \ldots, X_{j_{d}}\right\} \in K} f\left(X_{j_{1}}, \ldots, X_{j_{d}}\right) \\
& =\sum_{l_{1}, \ldots, l_{s}=0}^{\infty} f_{l_{1} \ldots l_{s}} n^{-d / 2} \sum_{d\left(\bar{k}_{u}, \bar{k}_{v}\right)>m, 1 \leqslant u \neq v \leqslant s} e_{l_{1}}^{(1)}\left(\bar{X}_{\bar{k}_{1}}\right) \cdots e_{l_{s}}^{(s)}\left(\bar{X}_{\bar{k}_{s}}\right) \\
& =\sum_{l_{1}, \ldots, l_{s}=0}^{N} f_{l_{1} \ldots l_{s}} n^{-d / 2} \sum_{d\left(\bar{k}_{u}, \bar{k}_{v}\right)>m, 1 \leqslant u \neq v \leqslant s} e_{l_{1}}^{(1)}\left(\bar{X}_{\bar{k}_{1}}\right) \cdots e_{l_{s}}^{(s)}\left(\bar{X}_{\bar{k}_{s}}\right) \\
& \quad+\sum_{\max \left(l_{1}, \ldots, l_{s}\right)>N} f_{l_{1} \ldots l_{s}} n^{-d / 2} \sum_{d\left(\bar{k}_{u}, \bar{k}_{v}\right)>m, 1 \leqslant u \neq v \leqslant s} e_{l_{1}}^{(1)}\left(\bar{X}_{\bar{k}_{1}}\right) \cdots e_{l_{s}}^{(s)}\left(\bar{X}_{\bar{k}_{s}}\right) .
\end{aligned}
$$


В случае наличия такого $T_{u}$, что $\#\left(T_{u}\right) \geqslant 3$, из разложения величины $(24)$ в сумму произведений (25) и рассуждений после введения этих формул следует, что первое слагаемое в правой части (32) сходится к нулю по вероятности. В ситуации с одно- и двухэлементными блоками, применив лемму 1 , нетрудно видеть, что

$$
\begin{aligned}
& \sum_{l_{1}, \ldots, l_{s}=0}^{N} f_{l_{1} \ldots l_{s}} n^{-d / 2} \sum_{d\left(\bar{k}_{u}, \bar{k}_{v}\right)>m, 1 \leqslant u \neq v \leqslant s} e_{l_{1}}^{(1)}\left(\bar{X}_{\bar{k}_{1}}\right) \cdots e_{l_{s}}^{(s)}\left(\bar{X}_{\bar{k}_{s}}\right) \\
& \stackrel{d}{\longrightarrow} \sum_{i_{1}, \ldots, i_{s_{1}}=1}^{N} f_{i_{1} \ldots i_{s_{1}}}^{(K)} \sum_{q=0}^{\left[s_{1} / 2\right]}(-1)^{q} \sum_{p \in I\left(i_{1}, \ldots, i_{s_{1}}, q\right)} M_{p} \prod_{i \in p^{c}} \tau_{i} .
\end{aligned}
$$

Докажем, что при $N \rightarrow \infty$ второе слагаемое в правой части (32) сходится к нулю в среднеквадратичном для любого класса, содержащего не менее $[d / 2]+1$ неделимых блоков. Для удобства записи будем обозначать $\bar{X}^{(i)}$ различные выборки из класса $K$ :

$$
\begin{gathered}
\mathbf{E}\left(n^{-d / 2} \sum_{\max \left(l_{1}, \ldots, l_{s}\right)>N} f_{l_{1} \ldots l_{s}} \sum_{d\left(\bar{k}_{u}, \bar{k}_{v}\right)>m, 1 \leqslant u \neq v \leqslant s} e_{l_{1}}^{(1)}\left(\bar{X}_{\bar{k}_{1}}\right) \cdots e_{l_{s}}^{(s)}\left(\bar{X}_{\bar{k}_{s}}\right)\right)^{2} \\
=n^{-d} \sum_{\bar{X}^{(1)}, \bar{X}^{(2)} \in K} \sum_{\max \left\{l_{k}\right\}, \max \left\{i_{k}\right\}>N} f_{l_{1} \ldots l_{s}} f_{i_{1} \ldots i_{s}} \mathbf{E} e_{l_{1}}^{(1)}\left(\bar{X}_{\bar{k}_{1}}^{(1)}\right) \\
\cdots e_{l_{s}}^{(s)}\left(\bar{X}_{\bar{k}_{s}}^{(1)}\right) e_{i_{1}}^{(1)}\left(\bar{X}_{\bar{k}_{1}^{\prime}}^{(2)}\right) \cdots e_{i_{s}}^{(s)}\left(\bar{X}_{\bar{k}_{s}^{\prime}}^{(2)}\right)
\end{gathered}
$$

Внесение знака математического ожидания под знак ряда здесь законно в силу сходимости соответствующих рядов в среднеквадратичном.

Заметим, что если в классе $s \geqslant[d / 2]+1$ неделимых блоков, то среди них по крайней мере $2 s-d$ одноэлементных. Следовательно, среди блоков $\bar{X}_{\bar{k}_{1}}^{(1)}, \ldots, \bar{X}_{\bar{k}^{\prime}}^{(2)}$ как минимум $4 s-2 d$ одноэлементных, или, иначе, не более $2 d-2 s$ блоков большей длины. Если хотя бы один из одноэлементных блоков находится на расстоянии, большем, чем $m$, от всех остальных, то математическое ожидание в правой части (33) будет равно нулю (независимо от индексов $\left.l_{1}, \ldots, l_{s}, i_{1}, \ldots, i_{s}\right)$. Значит, для того, чтобы слагаемые отличались от нуля, каждый одноэлементный блок должен находиться на расстоянии, не превышающем $m$, либо от другого одноэлементного блока, либо от блока большей длины. Способов выбрать $\bar{X}^{(1)}$ и $\bar{X}^{(2)}$, удовлетворяющие таким условиям, не больше

$$
C(d, m) n^{2 d-2 s} n^{2 s-d} \leqslant C(d, m) n^{d},
$$

где $C(d, m)$ - константа, зависящая только от $d$ и $m$. А для фиксированных $\bar{X}^{(1)}$ и $\bar{X}^{(2)}$

$$
\mathbf{E} \sum_{\max \left\{l_{k}\right\}, \max \left\{i_{k}\right\}>N} f_{l_{1} \ldots l_{s}} f_{i_{1} \ldots i_{s}} e_{l_{1}}^{(1)}\left(\bar{X}_{\bar{k}_{1}}^{(1)}\right) \cdots e_{l_{s}}^{(s)}\left(\bar{X}_{\bar{k}_{s}}^{(1)}\right) e_{i_{1}}^{(1)}\left(\bar{X}_{\bar{k}_{1}^{\prime}}^{(2)}\right) \ldots e_{i_{s}}^{(s)}\left(\bar{X}_{\bar{k}_{s}^{\prime}}^{(2)}\right)
$$




$$
\begin{aligned}
\leqslant & {\left[\mathbf{E}\left(\sum_{\max \left\{l_{k}\right\}>N} f_{l_{1} \ldots l_{s}} e_{l_{1}}^{(1)}\left(\bar{X}_{\bar{k}_{1}}^{(1)}\right) \cdots e_{l_{s}}^{(s)}\left(\bar{X}_{\bar{k}_{s}}^{(1)}\right)\right)^{2}\right]^{1 / 2} } \\
& \times\left[\mathbf{E}\left(\sum_{\max \left\{i_{k}\right\}>N} f_{i_{1} \ldots i_{s}} e_{i_{1}}^{(1)}\left(\bar{X}_{\bar{k}_{1}^{\prime}}^{(2)}\right) \cdots e_{i_{s}}^{(s)}\left(\bar{X}_{\bar{k}_{s}^{\prime}}^{(2)}\right)\right)^{2}\right]^{1 / 2} \\
= & \sum_{\max \left\{l_{k}\right\}>N} f_{l_{1} \ldots l_{s}}^{2} .
\end{aligned}
$$

А значит,

$$
\begin{aligned}
& \mathbf{E}\left(n^{-d / 2} \sum_{\max \left(l_{1}, \ldots, l_{s}\right)>N} f_{l_{1} \ldots l_{s}} \sum_{d\left(\bar{k}_{u}, \bar{k}_{v}\right)>m, 1 \leqslant u \neq v \leqslant s} e_{l_{1}}^{(1)}\left(\bar{X}_{\bar{k}_{1}}\right) \cdots e_{l_{s}}^{(s)}\left(\bar{X}_{\bar{k}_{s}}\right)\right)^{2} \\
& \quad \leqslant C(d, m) \sum_{\max \left\{l_{k}\right\}>N} f_{l_{1} \ldots l_{s}}^{2} \rightarrow 0
\end{aligned}
$$

при $N \rightarrow \infty$, что и требовалось доказать.

Теперь покажем, что при $N \rightarrow \infty$ «хвост» предельной величины в (31) сходится к нулю в среднеквадратичном.

Пусть $\left\{i_{1}^{\prime \prime}, \ldots, i_{s_{1}}^{\prime \prime}\right\}-$ некоторая перестановка $\left\{i_{1}^{\prime}, \ldots, i_{s_{1}}^{\prime}\right\}$. Для начала докажем, что

$$
\sum_{\max \left\{i_{k}\right\}, \max \left\{i_{k}^{\prime}\right\}>N}\left|f_{i_{1} \ldots i_{s_{1}}} f_{i_{1}^{\prime} \ldots i_{s_{1}}^{\prime}}\right|\left|M_{i_{1} i_{1}^{\prime \prime}} \cdots M_{i_{s_{1}} i_{s_{1}}^{\prime \prime}}\right| \rightarrow 0
$$

при коэффициентах $f_{i_{1} \ldots i_{s_{1}}}$, суммируемых в квадрате. Ввиду определения $M_{k l}$ достаточно для произвольных $j_{1}, \ldots, j_{s_{1}}$ показать, что

$$
\begin{gathered}
\sum_{i_{1}, \ldots, i_{s_{1}}, i_{1}^{\prime}, \ldots, i_{s_{1}}^{\prime}=1}^{\infty}\left|f_{i_{1} \ldots i_{s_{1}}} f_{i_{1}^{\prime} \ldots i_{s_{1}}^{\prime}}\right|\left|\mathbf{E} e_{i_{1}}\left(X_{1}\right) e_{i_{1}^{\prime \prime}}\left(X_{j_{1}+1}\right)\right| \\
\ldots\left|\mathbf{E} e_{i_{s_{1}}}\left(X_{1}\right) e_{i_{s_{1}}^{\prime \prime}}\left(X_{j_{s_{1}}+1}\right)\right|<\infty .
\end{gathered}
$$

Сначала зафиксируем все индексы, кроме $i_{s_{1}}, i_{s_{1}}^{\prime \prime}$, и проведем суммирование только по последним:

$$
\begin{aligned}
\mid \mathbf{E} e_{i_{1}} & \left(X_{1}\right) e_{i_{1}^{\prime \prime}}\left(X_{j_{1}+1}\right)|\cdots| \mathbf{E} e_{i_{s_{1}-1}}\left(X_{1}\right) e_{i_{s_{1}-1}^{\prime \prime}}\left(X_{j_{s_{1}-1}+1}\right) \mid \\
& \times \sum_{i_{s_{1}}, i_{s_{1}}^{\prime \prime}=1}^{\infty}\left|f_{i_{1} \ldots i_{s_{1}}} f_{i_{1}^{\prime} \ldots i_{s_{1}}^{\prime}}\right| \mathbf{E} e_{i_{s_{1}}}\left(X_{1}\right) e_{i_{s_{1}}^{\prime \prime}}\left(X_{j_{s_{1}}+1}\right) \mid \\
\leqslant & \left|\mathbf{E} e_{i_{1}}\left(X_{1}\right) e_{i_{1}^{\prime \prime}}\left(X_{j_{1}+1}\right)\right| \cdots\left|\mathbf{E} e_{i_{s_{1}-1}}\left(X_{1}\right) e_{i_{s_{1}-1}^{\prime \prime}}\left(X_{j_{s_{1}-1}+1}\right)\right| \\
& \times\left(\sum_{i_{s_{1}}=1}^{\infty} f_{i_{1} \ldots i_{s_{1}}}^{2}\right)^{1 / 2}\left(\sum_{i_{s_{1}}^{\prime \prime}=1}^{\infty} f_{i_{1}^{\prime} \ldots i_{s_{1}}^{\prime}}^{2}\right)^{1 / 2} .
\end{aligned}
$$


Аналогично, суммируя последовательно по каждой паре индексов и учитывая, что $\left\{i_{1}^{\prime \prime}, \ldots, i_{s_{1}}^{\prime \prime}\right\}=\left\{i_{1}^{\prime}, \ldots, i_{s_{1}}^{\prime}\right\}$, получаем

$\sum_{i_{1}, \ldots, i_{s_{1}}, i_{1}^{\prime}, \ldots, i_{s_{1}}^{\prime}=1}^{\infty}\left|f_{i_{1} \ldots i_{s_{1}}} f_{i_{1}^{\prime} \ldots i_{s_{1}}^{\prime}}\right|\left|M_{i_{1} i_{1}^{\prime \prime}} \cdots M_{i_{s_{1}} i_{s_{1}}^{\prime \prime}}\right| \leqslant(2 m+1)^{s_{1}} \sum_{i_{1}, \ldots, i_{s_{1}}=1}^{\infty} f_{i_{1} \ldots i_{s_{1}}}^{2}$.

Запишем второй момент интересующего нас хвоста предельного ряда в правой части (31):

$$
\begin{aligned}
& \sum_{\max \left\{i_{k}\right\}, \max \left\{i_{k}^{\prime}\right\}>N} f_{i_{1} \ldots i_{s_{1}}} f_{i_{1}^{\prime} \ldots i_{s_{1}}^{\prime}} \sum_{q, q^{\prime}=0}^{\left[s_{1} / 2\right]}(-1)^{q+q^{\prime}} \\
& \times \sum_{p \in I\left(i_{1}, \ldots, i_{s_{1}}, q\right)} \sum_{p^{\prime} \in I\left(i_{1}^{\prime}, \ldots, i_{s_{1}}^{\prime}, q^{\prime}\right)} M_{p} M_{p^{\prime}} \mathbf{E} \prod_{i \in p^{c}} \tau_{i} \prod_{i^{\prime} \in p^{\prime c}} \tau_{i^{\prime}} \\
= & \sum_{\max \left\{i_{k}\right\}, \max \left\{i_{k}^{\prime}\right\}>N} f_{i_{1} \ldots i_{s_{1}}} f_{i_{1}^{\prime} \ldots i_{s_{1}}^{\prime}} \Sigma^{*} .
\end{aligned}
$$

Покажем, что

$$
\Sigma^{*}=\sum_{\left\{i_{1}^{\prime \prime}, \ldots, i_{s_{1}}^{\prime \prime}\right\}} M_{i_{1} i_{1}^{\prime \prime}} \ldots M_{i_{s_{1}} i_{s_{1}}^{\prime \prime}}
$$

где суммирование в правой части проводится по всем различным перестановкам $\left\{i_{1}^{\prime}, \ldots, i_{s_{1}}^{\prime}\right\}$. Воспользуемся представлением смешанных моментов гауссовской последовательности через элементы ковариационной матрицы [3, п. 1.3]:

$$
\mathbf{E} \prod_{i \in p^{c}} \tau_{i} \prod_{i^{\prime} \in p^{\prime c}} \tau_{i^{\prime}}=\sum_{p_{0} \in I\left(p^{c}, p^{\prime c}, 2 s_{1}-2 q-2 q^{\prime}\right)} M_{p_{0}},
$$

таким образом,

$$
\Sigma^{*}=\sum_{q, q^{\prime}=0}^{\left[s_{1} / 2\right]}(-1)^{q+q^{\prime}} \sum_{p \in I\left(i_{1}, \ldots, i_{s_{1}}, q\right)} \sum_{p^{\prime} \in I\left(i_{1}^{\prime}, \ldots, i_{s_{1}}, q^{\prime}\right)} \sum_{p_{0} \in I\left(p^{c}, p^{\prime c}, 2 s_{1}-2 q-2 q^{\prime}\right)} M_{p} M_{p^{\prime}} M_{p_{0}} .
$$

Очевидно, правая часть (34) соответствует в вышеприведенной сумме части слагаемых при $q=0$ и $q^{\prime}=0$, так как слагаемые этой правой части составлены из пар, в которых один индекс принадлежит $\left\{i_{1}, \ldots, i_{s_{1}}\right\}$, а другой $\left\{i_{1}^{\prime}, \ldots, i_{s_{1}}^{\prime}\right\}$. Значит, эти слагаемые не могут содержать в качестве сомножителя $M_{p}$ или $M_{p^{\prime}}$ с соответствующими $q \geqslant 1$ или $q^{\prime} \geqslant 1$. Рассмотрим слагаемые при $q=0$ и $q^{\prime}=0$, не входящие в правую часть (34). Очевидно, они соответствуют всевозможным $p_{0} \in I\left(i_{1}, \ldots, i_{s_{1}}, i_{1}^{\prime}, \ldots, i_{s_{1}}^{\prime}, 2 s_{1}\right)$, содержащим хотя бы одну пару, в которой оба индекса принадлежат $\left\{i_{1}, \ldots, i_{s_{1}}\right\}$ или $\left\{i_{1}^{\prime}, \ldots, i_{s_{1}}^{\prime}\right\}$. Обозначим все такие пары $p_{1}, \ldots, p_{N}$, где $N=2 C_{s_{1}}^{2}=s_{1}\left(s_{1}-1\right)$. Имеем

$$
\sum_{p_{0} \in I\left(i_{1}, \ldots, i_{s_{1}}^{\prime}, 2 s_{1}\right): p_{j} \in p_{0}, 1 \leqslant j \leqslant N} M_{p_{0}} .
$$


Заметим, что если различные пары $p_{k}$ и $p_{l}$ имеют один общий индекс, то фиксированное $p_{0}$ может содержать лишь одну из них. Используя формулу включения-исключения, а также формулу для математического ожидания произведения гауссовских величин, можно записать введенную сумму следующим образом:

$$
\begin{aligned}
& \sum_{j=1}^{N} M_{p_{j}} \mathbf{E} \prod_{i \in\left\{i_{1}, \ldots, i_{s_{1}}^{\prime}\right\} \backslash p_{j}} \tau_{i}-\sum_{k<l: p_{k} \cap p_{l}=\varnothing} M_{p_{k}} M_{p_{l}} \mathbf{E} \prod_{i \in\left\{i_{1}, \ldots, i_{s_{1}}^{\prime}\right\} \backslash\left(p_{k} \cup p_{l}\right)} \tau_{i} \\
& +\cdots-\sum_{k_{1}<\cdots<k_{2\left[s_{1} / 2\right]}: p_{k_{i}} \cap p_{k_{j}}=\varnothing, i \neq j} M_{p_{k_{1}}} \cdots M_{p_{k_{2\left[s_{1} / 2\right]}}} \\
& \times \mathbf{E} \prod_{i \in\left\{i_{1}, \ldots, i_{s_{1}}^{\prime}\right\} \backslash\left(p_{k_{1}} \cup \cdots \cup p_{k_{2\left[s_{1} / 2\right]}}\right)} \tau_{i} .
\end{aligned}
$$

Нетрудно заметить, что предложенное разложение в точности совпадает с тем фрагментом суммы $-\Sigma^{*}$, где хотя бы одно из $q$ и $q^{\prime}$ больше нуля. Это доказывает равенство (34), а учитывая рассуждения выше, и сходимость предельного ряда в среднеквадратичном. Лемма доказана.

Всевозможные $\left\{X_{j_{1}}, \ldots, X_{j_{d}}\right\}$ делятся на конечное число классов эквивалентности, зависящее только от $d$ и $m$, следовательно, $V$ статистику можно разбить на конечное число слагаемых, каждое из которых является нормированной суммой $f\left(X_{j_{1}}, \ldots, X_{j_{d}}\right)$ по векторам наблюдений из некоторого фиксированного класса.

Для классов, в которых не менее $[d / 2]+1$ неделимых блоков, слабый предел получен в лемме 2 . Для классов, в которых менее $d / 2$ или ровно $d / 2$ (для четных $d$ ) неделимых блоков, пределы (по вероятности) выписаны соответственно в (16) и (14).

В силу рассуждений в начале доказательства теоремы, для перехода к $V$-статистике можно просуммировать по всем соответствующим классам предельные выражения в правых частях (31) и (14). Теорема 1 доказана.

Д ок азат е ль с т в о т е о ре м ы 2. Доказательство по сути повторяет доказательство теоремы 1 за тем исключением, что в случае $U$-статистик мы не рассматриваем классы векторов с повторяющимися элементами. Отсюда закономерно следует, что в двухэлементных блоках расстояние между индексами наблюдений не должно равняться 0 , что и отражено в выражениях для $\sum_{K \in \mathfrak{K}_{1}^{(d-2 k)}}$ и $\sum_{K^{\prime} \in \mathfrak{K}_{1}^{(0)}}$.

Д ок аз а т е ль с т в о з а м е ч а и я 2. Докажем сначала совпадение пределов для статистик Мизеса при выполнении условий обеих интересующих нас в этом случае теорем.

Нетрудно заметить, что слагаемое при $k=0$ (которому соответствует единственный класс $K)$ и $q=0$ в правой части (21) совпадает с правой частью (10). Достаточно показать, что при выполнении условий теоремы 2 из [2] оставшаяся сумма равна 0. 
Упомянутые условия, как было сказано, обеспечивают сходимость частичных сумм ряда (4) к ядру почти наверное относительно совместного распределения $X_{j_{1}}, \ldots, X_{j_{d}}$ для любых (в том числе и для совпадающих) $X_{j_{1}}, \ldots, X_{j_{d}}$ из исходной стационарной последовательности. Таким образом,

$$
\begin{aligned}
f_{i_{1} \ldots i_{d-2 k}}^{(K)}= & \sum_{i_{d-2 k+1}, \ldots, i_{d}=1}^{\infty} f_{\left(i_{1} \ldots i_{d}\right)} \mathbf{E} e_{i_{l_{1}}}\left(X_{m}\right) e_{i_{l_{2}}}\left(X_{m+m_{1}}\right) \\
& \times \cdots \times \mathbf{E} e_{i_{l_{2 k-1}}}\left(X_{m}\right) e_{i_{l_{2 k}}}\left(X_{m+m_{k}}\right),
\end{aligned}
$$

где в перестановке $\left(i_{1} \ldots i_{d}\right)$ индексы $i_{d-2 k+1}, \ldots, i_{d}$ занимают места $k_{d-2 k+1}, \ldots, k_{d}$ из $(23)$ по порядку, а на остальных местах по порядку расположены $i_{1}, \ldots, i_{d-2 k}$. Множество индексов $\left\{l_{1}, \ldots, l_{2 k}\right\}$ совпадает с множеством $\{d-2 k+1, \ldots, d\}$, и $\left\{\left\{k_{l_{1}}, k_{l_{2}}\right\}, \ldots,\left\{k_{l_{2 k-1}}, k_{l_{2 k}}\right\}\right\}=p_{1}\left(p_{1}\right.$ задано в $(23))$. При фиксированных $i_{1}, \ldots, i_{d-2 k}$ просуммируем $f_{i_{1} \ldots i_{d-2 k}}^{(K)}$ по классам $K$ с одинаковыми $p_{1}$ из $(23)$, но с различными интервалами $m_{i}$ :

$$
\sum_{i_{d-2 k+1}, \ldots, i_{d}=1}^{\infty} f_{\left(i_{1} \ldots i_{d}\right)} M_{i_{l_{1}} i_{l_{2}}} \cdots M_{i_{l_{2 k-1} i_{2 k}}} .
$$

Зафиксируем индексы $i_{1}, \ldots, i_{d}$ и перестановку $\left(i_{1} \ldots i_{d}\right)$. Далее, выберем из нее $d-2 r$ индексов $(0<r<d / 2)$, занимающих конкретные $d-2 r$ мест в перестановке, которые будут соответствовать $p^{c}$. Приведем подобные слагаемые при каждом произведении $f_{\left(i_{1} \ldots i_{d}\right)} \prod_{i \in p^{c}} \tau_{i}$ в $(21)$, используя преобразования выше.

Переобозначим индексы $\left\{i_{1}, \ldots, i_{d}\right\} \backslash p^{c}$ как $\left\{j_{1}, \ldots, j_{2 r}\right\}$. Из них некоторые $2 k$ индексов $(0 \leqslant k \leqslant r)$ соответствуют двухэлементным блокам из $(23)$, а оставшиеся $2(r-k)=2 q-$ парам из $(21)$.

Чтобы просуммировать интересующие нас подобные слагаемые по $k$ и классам $K$, нужно из $2 r$ индексов выбрать $2 k$ для двухэлементных блоков и обе части разбить на пары. В результате получаем:

$$
\begin{aligned}
& \sum_{k=0}^{r}(-1)^{r-k} \sum_{\left\{j_{1}^{\prime}, \ldots, j_{2 k}^{\prime}\right\} \subset\left\{j_{1}, \ldots, j_{2 r}\right\}} \sum_{p, p_{1}} M_{p} M_{p_{1}} \\
& =\sum_{\tilde{p} \in I\left(j_{1}, \ldots, j_{2 r}, r\right)} M_{\tilde{p}} \sum_{k=0}^{r} C_{r}^{k} 1^{k}(-1)^{r-k}=0 .
\end{aligned}
$$

Здесь

$$
p_{1} \in I\left(j_{1}^{\prime}, \ldots, j_{2 k}^{\prime}, k\right), \quad p \in I\left(\left\{j_{1}, \ldots, j_{2 r}\right\} \backslash\left\{j_{1}^{\prime}, \ldots, j_{2 k}^{\prime}\right\}, r-k\right) .
$$

В случае четного $d$ при $r=d / 2$ (т.е. при $p^{c}=\varnothing$ ) аналогичным образом доказывается, что все постоянные слагаемые из $(21)$, включая $C$, также дают в сумме 0. 
Теперь докажем совпадение пределов $U$-статистик в случае выполнения как условий теоремы 2, так и условий теоремы 1 из [2]. Эти условия, как и прежде, по сути дают возможность представить коэффициент $f_{i_{1}, \ldots, i_{d-2 k}}^{(K)}$ в виде $(35)$.

Введем обозначения. Если $p=\left\{\left\{i_{l_{1}}, i_{l_{2}}\right\}, \ldots,\left\{i_{l_{2 q-1}}, i_{l_{2 q}}\right\}\right\}$, то будем обозначать

$$
\begin{gathered}
\delta_{p}=\delta_{i_{l_{1}} i_{l_{2}}} \cdots \delta_{i_{l_{2 q-1} i_{l_{2 q}}}}, \\
\widetilde{M}_{p}=\left(M_{i_{l_{1}} i_{l_{2}}}-\delta_{i_{l_{1}} i_{l_{2}}}\right) \cdots\left(M_{i_{l_{2 q-1}} i_{l_{2 q}}}-\delta_{i_{l_{2 q-1}} i_{l_{2 q}}}\right) .
\end{gathered}
$$

Если в правой части (22) в слагаемых при $k=0$ для каждых $q$ и $p$ заменить $M_{p}$ на $\delta_{p}$, то учитывая замечание 3 , получим правую часть (9). Докажем, что разность правых частей (22) и (9) равна нулю.

Рассуждаем таким же образом, как и раньше. Фиксируем перестановку $\left(i_{1} \ldots i_{d}\right)$ и $d-2 r$ индексов из нее для $p^{c}(1<r<d / 2)$ и приводим подобные слагаемые при $f_{\left(i_{1} \ldots i_{d}\right)} \prod_{i \in p^{c}} \tau_{i}$. Так же обозначаем $\left\{j_{1}, \ldots, j_{2 r}\right\}=\left\{i_{1}, \ldots, i_{d}\right\} \backslash p^{c}$. Получаем множитель при $f_{\left(i_{1} \ldots i_{d}\right)} \prod_{i \in p^{c}} \tau_{i}$ (в интересующей нас разности правых частей):

$$
\sum_{k=1}^{r}(-1)^{r-k} \sum_{\left\{j_{1}^{\prime}, \ldots, j_{2 k}^{\prime}\right\} \subset\left\{j_{1}, \ldots, j_{2 r}\right\}} \sum_{p, p_{1}} M_{p} \widetilde{M}_{p_{1}}+(-1)^{r} \sum_{p \in I\left(j_{1}, \ldots, j_{2 r}, r\right)}\left(M_{p}-\delta_{p}\right) .
$$

Здесь $p_{1} \in I\left(j_{1}^{\prime}, \ldots, j_{2 k}^{\prime}, k\right), p \in I\left(\left\{j_{1}, \ldots, j_{2 r}\right\} \backslash\left\{j_{1}^{\prime}, \ldots, j_{2 k}^{\prime}\right\}, r-k\right)$, а $\widetilde{M}_{p_{1}}$ появляется за счет того, что в двухэлементных блоках не может быть одинаковых индексов, в отличие от ситуации со статистиками Мизеса. Последняя сумма в (36) соответствует разности при $k=0$, согласно сделанному выше замечанию.

Остается показать, что выражение в (36) есть тождественный нуль. Легко видеть, что $p \cup p_{1}=\widetilde{p} \in I\left(j_{1}, \ldots, j_{2 r}, r\right)$. Зафиксируем некое $\widetilde{p}$, т.е. будем рассматривать такие слагаемые из (36), в которых $p \cup p_{1}=\widetilde{p}$. Пусть $0 \leqslant v \leqslant r$ и $p_{2}=\left\{\left\{j_{l_{1}}, j_{l_{2}}\right\}, \ldots,\left\{j_{l_{2 v-1}}, j_{l_{2 v}}\right\}\right\} \subset \widetilde{p}$. Если раскрыть скобки в выражении для $\widetilde{M}_{p_{1}}$, то интересующие нас слагаемые представимы в виде суммы различных $M_{p_{2}}$, умноженных на степени -1 и произведение символов Кронекера.

Рассмотрим сначала $1 \leqslant v \leqslant r-1$ и приведем подобные слагаемые при $M_{p_{2}}$ (в предположении $p \cup p_{1}=\widetilde{p}$ ). Здесь $k$ может принимать значения от $r-v$ до $r$, при этом среди $v$ пар, образующих $p_{2}$, какие-то $r-k$ образуют $p$, а остальные относятся к $p_{1}$. Таким образом, следует учесть количество различных вариантов в этом случае. В результате, получаем множитель при $M_{p_{2}}$ :

$$
\sum_{k=r-v}^{r}(-1)^{r-k} \delta_{\tilde{p} / p_{2}}(-1)^{r-v} C_{v}^{r-k}=\delta_{\tilde{p} / p_{2}}(-1)^{r-v} \sum_{q=0}^{v}(-1)^{q} C_{v}^{q}=0 .
$$


Если $v=0$, т.е. $M_{p_{2}}=1$, то множитель состоит из двух слагаемых, при $k=r$ и $k=0$ :

$$
\delta_{\tilde{p}}(-1)^{r}+\delta_{\tilde{p}}(-1)^{r+1}=0 .
$$

В случае $v=r, M_{p_{2}}=M_{\tilde{p}}$, множитель выглядит как в случае статистик Мизеса:

$$
\sum_{k=1}^{r}(-1)^{r-k} C_{v}^{r-k}+(-1)^{r}=0 .
$$

Случай $r=d / 2$, если $d$ четно, разбирается аналогично. Замечание 2 доказано.

В заключение автор хотел бы поблагодарить И. С. Борисова за постановку задачи и ценные замечания, а также рецензента, указавшего ряд неточностей в первоначальной версии работы.

\section{СПИСОК ЛИТЕРАТУРЫ}

1. Борисов И.С., Быстров А.А. Предельные теоремы для канонических статистик Мизеса, построенных по зависимым наблюдениям. - Сиб. матем. журн., 2006, т. 47, № 6, с. 1205-1217.

2. Борисов И. С., Володько Н. В. Ортогональные ряды и предельные теоремы для канонических $U$ - и $V$-статистик от стационарно связанных наблюдений. - Матем. труды, 2008, т. 11, № 1, с. 25-48.

3. Гихман И. И., Скороход А.В. Введение в теорию случайных процессов. М.: Наука, 1965,655 с.

4. Колмогоров A.H., Фомин С.В. Элементы теории функций и функционального анализа. М.: Наука, 1968, 496 с.

5. Королюк В.С., Боровских Ю.В. Теория $U$-статистик. Киев: Наукова думка, $1989,383 \mathrm{c}$.

6. Утев С.A. Суммы случайных величин с $\varphi$-перемешиванием. - Труды Ин-та математики СО АН СССР, 1989, т. 13, с. 78-100.

7. Borisov I. S., Volodko N. V. Limit theorems and exponential inequalities for canonical $U$ - and $V$-statistics of dependent trials. - High Dimensional Probability V: The Luminy Volume. Ed. by C. Houdré et al. Beachwood: IMS, 2009, p. 108-130. (IMS Collections, v. 5.)

8. Eagleson G. K. Orthogonal expansions and U-statistics. - Austral. J. Statist., 1979, v. 21, № 3 , p. 221-237.

9. Hoeffding $W$. A class of statistics with asymptotically normal distribution. - Ann. Math. Statist., 1948, v. 19, p. 293-325.

10. von Mises $R$. On the asymptotic distribution of differentiable statistical functions. Ann. Math. Statist., 1947, v. 18, p. 309-348.

11. Rosenthal H. P. On the subspaces of $L^{p}(p>2)$ spanned by sequences of independent random variables. - Israel J. Math., 1970, v. 8, p. 273-303.

12. Rubin H., Vitale R. Asymptotic distribution of symmetric statistics. - Ann. Statist., 1980 , v. 8 , № 1, p. 165-170. 